\title{
A scaling analysis of the snake lightness illusion
}

\author{
Alexander D. Logvinenko \\ Glasgow Caledonian University, Glasgow, Scotland \\ Karin Petrini \\ University of Padua, Padua, Italy \\ AND \\ LaURenCe T. Maloney \\ New York University, New York, New York
}

\begin{abstract}
Logvinenko and Maloney (2006) measured perceived dissimilarities between achromatic surfaces placed in two scenes illuminated by neutral lights that could differ in intensity. Using a novel scaling method, they found that dissimilarities between light surface pairs could be represented as a weighted linear combination of two dimensions, "surface lightness" (a perceptual correlate of the difference in the logarithm of surface albedo) and "surface brightness" (which corresponded to the differences of the logarithms of light intensity across the scenes). Here we attempt to measure the contributions of these dimensions to a compelling lightness illusion (the "snake illusion"). It is commonly assumed that this illusion is a result of erroneous segmentation of the snake pattern into regions of unequal illumination. We find that the illusory shift in the snake pattern occurs along the surface lightness dimension, with no contribution from surface brightness. Thus, even if an erroneous segmentation of the snake pattern into strips of unequal illumination does happen, it reveals itself, paradoxically, as illusory changes in surface lightness rather than as surface brightness. We conjecture that the illusion strength depends on the balance between two groups of illumination cues signaling the true (uniform) illumination and the pictorial (uneven) illumination.
\end{abstract}

In the snake lightness illusion (Adelson, 2000; Somers \& Adelson, 1997) shown in Figure 1A, pairs of achromatic, diamond-shaped regions that have the same reflectance (albedo) take on very different appearances. This illusion serves to demonstrate how the lightness of surfaces of the same reflectance may differ dramatically, depending on the context within which they are presented. Well-known demonstrations of simultaneous lightness contrast confirm this point: The lightness assigned by the visual system to surfaces is affected by the surround of the surface.

In everyday scenes, though, objects do not markedly change their lightness when presented against different backgrounds. This phenomenon is sometimes referred to as lightness constancy with respect to background (Gilchrist, 2006; Whittle, 1994a, 1994b). Although simultaneous lightness contrast and lightness constancy have both been intensively studied for more than a century (for review, see Gilchrist, 2006), there is no clear understanding of why lightness constancy with respect to background holds in some circumstances but not in others.

Various models and theories of simultaneous lightness contrast have been put forward (for review, see Gilchrist, 2006). Many of them share the assumption that simultaneous lightness contrast is a by-product of another type of lightness constancy: lightness constancy with respect to illumination. It has been long recognized that, if lightness were based solely on the output of contrast mechanisms encoding the ratio of the luminance of surfaces to that of their surround (rather than the absolute luminance of surfaces), lightness perception would be independent of changes in illumination intensity (e.g., Cornsweet, 1970; Gilchrist, 2006; Whittle, 1994a, 1994b). As can be seen in Figure 1B, the snake illusion nearly disappears when the local contrasts of each target are equated. ${ }^{1}$ The appearance of the two configurations in Figures 1A and 1B is consistent with a local contrast rule: Targets of equal local contrast have equal lightness.

It must be borne in mind, however, that if lightness perception is determined exclusively by a local contrast rule, lightness constancy with respect to illumination can only be achieved at the cost of lightness constancy with respect to background. The theoretical significance of this contradiction is often minimized, as in the claim that the effect of simultaneous contrast is small and that in everyday scenes we usually do not notice that lightness constancy with respect to background is not perfect (Gilchrist, 2006; Whittle, 1994a, 1994b). Inspection of the snake and tile illusions, though, makes it evident that such failures need not be small. In particular, the effects are much stronger than those produced with simultaneous lightness contrast patterns. ${ }^{2}$

A. D. Logvinenko, a.logvinenko@gcal.ac.uk 
A

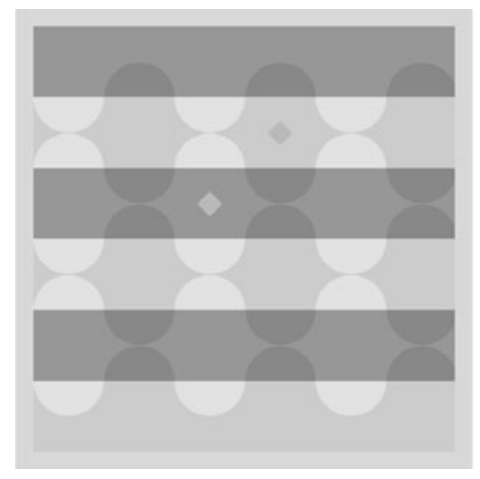

B

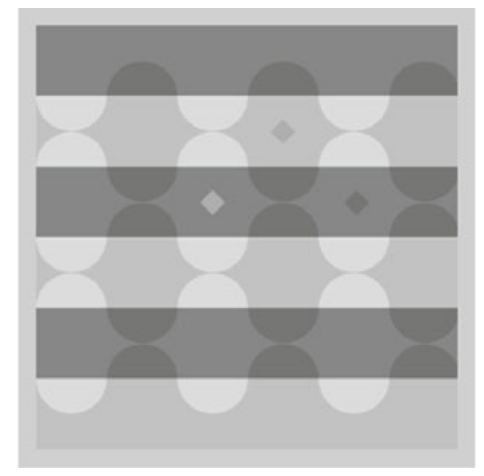

C

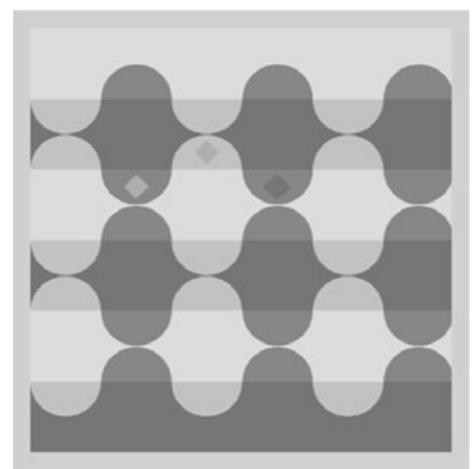

Figure 1. (A) Adelson's snake lightness illusion (Adelson, 2000; Somers \& Adelson, 1997). The two diamonds have the same reflectance (albedo) but appear to differ in lightness. (B) When the local contrast of the two diamonds in different strips of Adelson's snake pattern is equated (the two diamonds more to the right), the illusion disappears and the two diamonds are perceived as having almost the same lightness, although they have difference reflectances. (C) The "anti-snake" pattern of Logvinenko et al. (2005). The leftmost and middle diamonds have the same luminances but very different contrasts, yet they have almost the same lightness. The middle and rightmost diamonds have almost the same contrast but very different luminances; they differ markedly in lightness. The lightnesses of the diamonds in (A) and (B) are consistent with a local contrast rule, but not with a luminance ratio rule. The lightnesses of the diamonds in $(\mathrm{C})$ are not consistent with a local contrast rule, but are consistent with a luminance ratio rule.

At first glance, the reduction of the illusion with equicontrast targets (Figure 1B) supports low-level accounts of simultaneous lightness contrast (e.g., Cornsweet, 1970; Whittle, 1994a, 1994b) and the lightness perception theories that rest upon the local contrast rule (e.g., Blakeslee \& McCourt, 2003; Whittle, 2003). Indeed, in Figure 1B the targets of equal reflectance look quite different, whereas those differing in reflectance (thus luminance) but equal in contrast result in nearly equal lightness perceptions. However, as shown elsewhere (Logvinenko, Adelson, Ross, \& Somers, 2005), a minor spatial rearrangement of Figure 1A that leaves intact the reflectance of all the patches, as well as the targets' local contrast, drastically changes the targets' appearance (Figure 1C). Now the leftmost and middle targets have the same luminance and look almost the same, whereas the middle and rightmost targets equated for local contrast differ in lightness. In other words, in Figure 1C we observe nearly perfect lightness constancy with respect to background, and the appearance of surfaces is well predicted by what will be referred to as the luminance ratio rule: The lightnesses of targets are in the same proportion as their luminances. ${ }^{3}$ A particular case of this rule is that targets of equal luminance have equal lightness. ${ }^{4}$ The luminance ratio rule is consistent with lightness constancy with respect to background.

Although successful in accounting for perception of simple stimulus displays (such as a disc-annulus configuration), the local contrast rule does not generalize to more complex scenes, and does not always work out when targets are presented on different backgrounds. In other words, as Gilchrist et al. (1999) pointed out, local contrast is an ambiguous and incomplete cue to surface albedo. As a result, theories of lightness perception based on local contrast succeed in accounting for simultaneous lightness contrast, but fail to predict the everyday experience of lightness constancy with respect to background. The dilemma here is the dilemma noted by Gibson: One cannot use the same rule to predict veridical and illusory perception (Gibson, 1979).

Note that luminance ratio is equal to albedo ratio only within an area of equal illumination. The luminance ratio values in differently illuminated areas need to be brought to a "common denominator"; in other words, the luminance ratios are also ambiguous and need to be anchored (Gilchrist et al., 1999). This hypothetical process of anchoring implies that the visual system is capable of segmenting the illusion displays into regions of uniform illumination ("frameworks"; Gilchrist et al., 1999; Kardos, 1934; Katz, 1935). Although this problem has been debated for decades (Gilchrist, 2006; Gilchrist, Delman, \& Jacobsen, 1983), we still do not know how the visual system segments scenes into frameworks.

One possibility is that the visual system employs some geometrical and optical features of the luminance pattern to differentiate between luminance borders produced by reflectance and illumination edges that, in turn, can be used for segmentation of a scene into frameworks. More specifically, crossing over surface areas of different reflectance, a luminance border produced by illumination makes a series of $\mathrm{X}$-junctions in the luminance pattern (e.g., Adelson, 2000), with a constant luminance ratio along itself distinguishing it from a luminance border produced by a material (reflectance) change (e.g., Cavanagh \& Leclerc, 1989; Logvinenko, 2003; Marr, 1982). Moreover, illumination borders are usually fuzzier than reflectance borders, so, by analyzing the spatial frequency spectrum of a luminance border, the visual system may decide if it is produced by an illumination or by a material change (Land \& McCann, 1971). Also, illumination borders produce mainly luminance contrast at the border, whereas material borders typically involve chromatic as well as luminance contrast (e.g., Párraga, Troscianko, \& Tolhurst, 2000; Rubin \& Richards, 1982). 
None of these cues to an illumination border alone is necessary. For instance, shadows are known to be slightly bluer (e.g., Churma, 1994; Fine, MacLeod, \& Boynton, 2003); that is, shadow borders are usually accompanied by some chromatic contrast. A series of luminance $\mathrm{X}$-junctions with a constant luminance ratio can be produced by a reflectance pattern (as in Figure 1). Hence, the visual system must integrate multiple, competing illuminant cues (Maloney, 2002).

There is evidence that the visual system uses some builtin heuristics, based on the differences mentioned above, to classify luminance borders (as illumination or material). For example, it has been recently shown that straightness (vs. curvature) serves as a cue for an illumination border (Logvinenko et al., 2005). Kingdom (2003) provided elegant evidence that the visual system interprets edges with luminance and chromatic variations across them as reflectance borders, whereas those with only luminance variations are interpreted as illumination borders.

Once a luminance border is classified as an illumination border (i.e., a part of the border of a framework of equal illumination), an estimate of the luminance ratio across this border can be used by the visual system in evaluating the relative intensity of the illuminations in the two adjacent frameworks. This, in turn, allows evaluation of the relative reflectances in the whole scene.

We can frame the problem of estimating surface albedo in complex scenes, as follows. Let us consider a Mondrian-like achromatic pattern comprising $N$ homogeneous surfaces of various reflectances. Let us assume also that there are $n$ frameworks of equal illumination. In Figure 2, we show a simple example with four surfaces and two frameworks. As is typical in the study of lightness, the physical factors are most easily described in terms of the logarithm of surface albedos and the logarithms of light intensities. We will accordingly denote the logarithms of surface albedo of the $j$ th surface in the $i$ th framework as $R_{i}^{i}$, and the logarithms of the light intensities in the $i$ th framework as $E^{i}$. The logarithms, $L_{j}^{i}$, of the luminance of each of the $N$ surfaces are then

$$
\begin{aligned}
& L_{j}^{i}=R_{j}^{i}+E^{i}, \quad i=1, \ldots, n ; \\
& j=1, \ldots, k_{i} ; k_{1}+\cdots+k_{n}=N .
\end{aligned}
$$

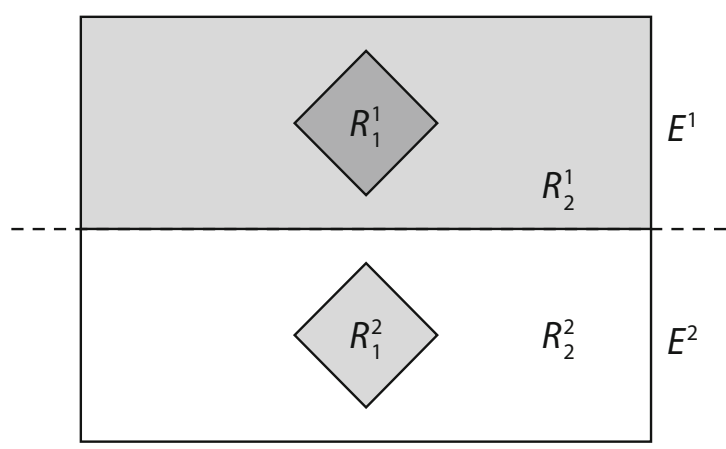

Figure 2. A simple Mondrian with four surfaces and two frameworks. See text for notation.
If the visual system is able to detect the illumination borders, and evaluate the luminance contrast across them, relative light intensities at the adjacent illumination borders (i.e., $E^{i+1}-E^{i}$ ) can be also evaluated. Therefore, we can assume that the terms $E^{i}$ in Equation 1 are known up to an arbitrary constant - say, $C$. In other words, the system of equations to be solved is now

$$
\begin{aligned}
& R_{j}^{i}+C=L_{j}^{i}-E^{i}, \quad i=1, \ldots, n \\
& j=1, \ldots, k_{i} ; k_{1}+\cdots+k_{n}=N,
\end{aligned}
$$

where $R_{j}^{i}$ and $C$ are unknown and $L_{j}^{i}$ and $E^{i}$ are known. Solving this system for each framework separately, one can uniquely evaluate the relative albedos.

Because of the constant $C$, the absolute values of both albedos $R_{j}^{i}$ and light intensities $E^{i}$ cannot be uniquely recovered. Indeed, $C$ can be arbitrarily split between the albedo and light intensities. Note, however, that it can be split between whole sets of $R_{j}^{i}$ and $E^{i}$, not among their individual members. If one of these sets is anchored, the others are uniquely determined from Equation 2. There are many observations showing that the lightness of an object may undergo an illusory change as a result of changing its apparent illumination (e.g., Koffka, 1935; Logvinenko, 2005; Logvinenko \& Menshikova, 1994). Such a tradeoff between lightness and apparent illumination emerges from the trade-off between the albedo and light intensity solutions of Equation 2.

Therefore, the patches of equal luminance $\left(L_{j}^{i}=L_{j}^{k}\right)$ should be assigned equal lightnesses only if they belong to the same framework (i.e., $E^{i}=E^{k}$ ). In other words, the luminance ratio rule is appropriate only within a framework, or between two identically illuminated frameworks. When patches of equal luminance belong to different frameworks (i.e., $E^{i} \neq E^{k}$ ) they will be assigned unequal lightnesses: The bigger the estimated illumination difference $E^{i}-E^{k}$, the larger the lightness difference. This fact is known in the visual literature as taking into account the illumination (e.g., Rock, 1975).

In the snake luminance pattern, the long horizontal edges are good candidates for illumination borders. Indeed, they are straight, and the luminance ratio across these edges is constant. Therefore, a plausible perceptual hypothesis may be that there are just two frameworks (i.e., $n=2$ ). In this case one can derive from Equation 2 the following equations:

$$
\begin{aligned}
& R_{1}^{1}+C=L_{1}^{1}-E^{1} ; \\
& R_{1}^{2}+C=L_{1}^{2}-E^{2},
\end{aligned}
$$

where $R_{1}^{1}$ and $R_{1}^{2}$ are the albedos of the diamond-shaped targets located in two adjacent strips; $L_{1}^{1}$ and $L_{1}^{2}$ are the target luminances; and $E^{1}$ and $E^{2}$ are the light intensities in these strips. Then we have

$$
R_{1}^{1}-R_{1}^{2}=L_{1}^{1}-L_{1}^{2}-\left(E^{1}-E^{2}\right) .
$$

Therefore, if the visual system assumes that $E^{1} \neq E^{2}$, the targets of equal luminance $\left(L_{1}^{1}=L_{1}^{2}\right)$ will be assigned unequal lightnesses, the lightness difference being proportional to the estimated illumination difference $E^{1}-E^{2}$. This explains the large illusory difference in lightness in 
Figures $1 \mathrm{~A}$ and $1 \mathrm{~B}$ between equiluminant targets, where the luminance ratio across the long horizontal border is 1.97, and almost no illusion in Figure $1 \mathrm{C}$, where the luminance ratio across the long horizontal border is close to 1.0 .

This hypothesis is plausible but, as it stands, evidently circular. We have no independent evidence that the visual system has effectively estimated that $E^{1} \neq E^{2}$ in Figure 1 . Moreover, it is possible that the visual system will treat a particular edge as partially due to a change in illumination and partially due to a change in surface material, particularly if cues are ambiguous. We return to this point in the General Discussion.

To test this hypothesis, we need to separately determine how the visual system interprets the long horizontal border in Figure 1 and similar configurations, and how it partitions $L_{1}^{1}-L_{1}^{2}$. To do so, we will use the method of Logvinenko and Maloney (2006) to separately estimate perceptual correlates of $E^{1}-E^{2}$ and $R_{1}^{1}-R_{1}^{2}$, which they refer to as "surface brightness" and "surface lightness," defined below. This information will allow us to determine whether the illusion is primarily an illusion of surface lightness, or of surface brightness, or of both together. If the visual system interprets the long horizontal borders in Figures 1A, B, and $\mathrm{C}$ as illumination rather than material (reflectance) borders, the dissimilarity judgments of regions of the snake pattern should be decomposable into separate contributions of surface lightness and surface brightness.

To verify such claims, the experimenter needs a method of assessing the separate contributions of illumination and albedo to lightness perception. Logvinenko and Maloney (2006) proposed and tested such a method based on dissimilarity scaling.

Logvinenko and Maloney (2006) used stimulus configurations consisting of two random arrangements ${ }^{5}$ of rectangular surface patches illuminated by lights that could differ in intensity (Figure 3A). We emphasize that, in their experiment, the subject saw actual matte surface patches illuminated by actual illuminants. The vertical edge in the center of Figure $3 \mathrm{~A}$ corresponds to a physical change in illumination intensity (illumination edge) in the scene viewed by the subject. Rather than have subjects make asymmetric matches across the two lighting environments, selecting a surface on the left half that matched a surface on the right half, Logvinenko and Maloney instead asked subjects to make a judgment $D$ of dissimilarity on a 30-point scale of pairs on surfaces in the left and right side designated by the experimenter. Ignoring a slight nonlinearity in response, they found that they could model responses as

$$
D=\delta_{E} \Delta E+\delta_{R} \Delta R \times \Gamma(E)+\varepsilon,
$$

where $\Delta E$ was the absolute value of the difference in the logarithms of light intensities, $\Delta R$ was the absolute value of the difference in the logarithms of surface albedos, and $\varepsilon$ represented independent, identically distributed Gaussian error. Observers perceive surface albedo differences as slightly greater under more intense lights, and the light expansion term $\Gamma(E)$ captures this effect. Fitting Equation 5 to the data, Logvinenko and Maloney found that $\delta_{R} / \delta_{E} \approx 4$,
A

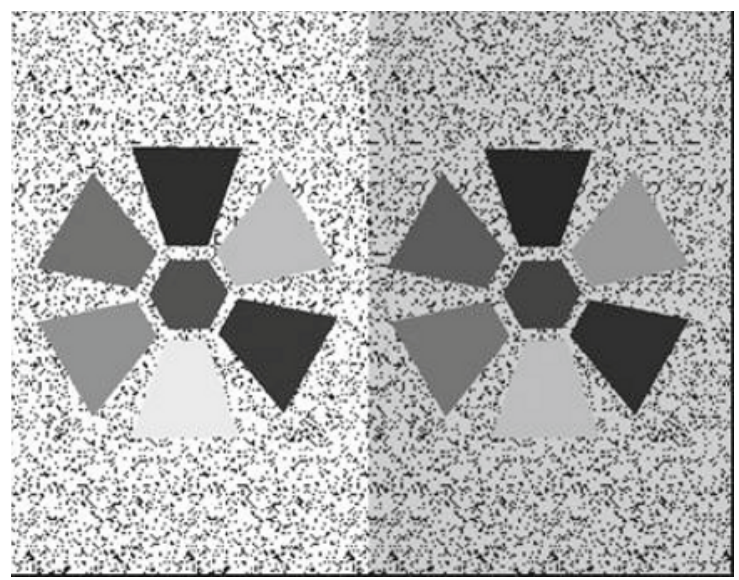

B

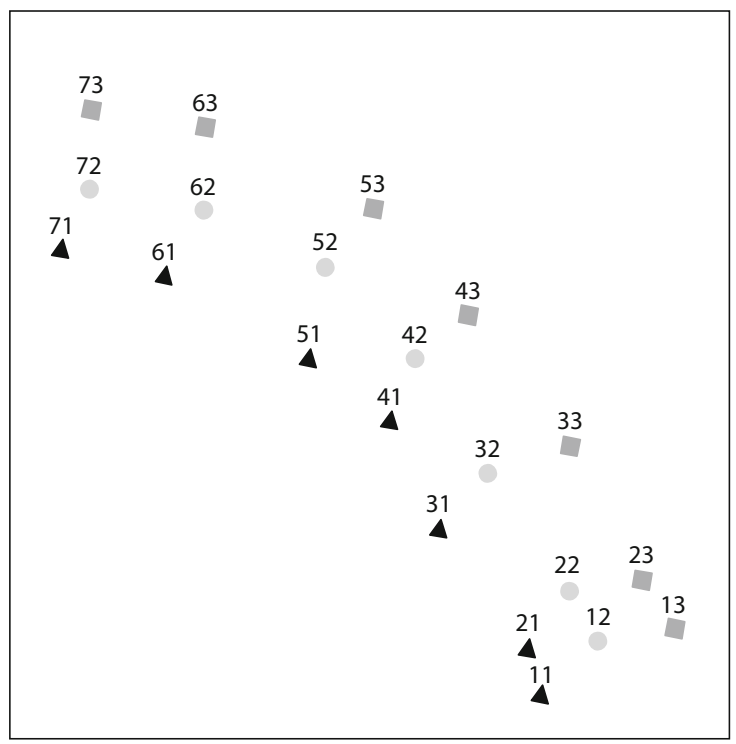

Figure 3. (A) Example of the stimulus configuration used in the experiment of Logvinenko and Maloney (2006). The observer saw two adjacent arrays of achromatic surfaces that could be illuminated by lights differing in intensity. When the lights differed in intensity, there was an evident vertical illumination edge in the center of the display as shown. (B) The nonmetric multidimensional scaling solution from Logvinenko and Maloney (2006). Surfaces under a single light fall close to arcs of concentric circles. The spacings between surfaces along a single arc correspond to differences in surface lightness. The spacings between arcs correspond to differences in surface brightness. See text.

indicating that a 16-fold decrease in light intensity was needed to produce the same effect as a twofold decrease in surface albedo, a kind of lightness constancy with respect to illumination framed entirely in terms of dissimilarity.

In Figure 3B, we show the nonmetric multidimensional scaling (MDS) solution (Cox \& Cox, 2001) corresponding to subjects' mean ratings in the experiment of Logvinenko 
and Maloney (2006). The MDS solution is an attempt to represent the rated dissimilarities as distances in a Euclidean space. The surfaces are regularly spaced along arcs of a circle separated by distances corresponding to changes in illumination. The increase in surface spacing with increased lighting is evident and corresponds to a shift of a circular arc inward or outward. ${ }^{6}$

An important feature of the output configuration in Figure $3 \mathrm{~B}$ is its two-dimensionality. Objects of different reflectance (albedo) illuminated by the same light lie on the same arc. Objects of the same reflectance (albedo) illuminated by different lights lie on the same radius. Light and surface differences, therefore, both contributed to dissimilarity judgments, and we could readily separate the contributions of light and surface. Logvinenko and Maloney (2006) have referred to these two dimensions as surface brightness and surface lightness.?

In this article, we apply the dissimilarity scaling approach of Logvinenko and Maloney (2006) to simple configurations such as the snake lightness illusion (Figure 1A). We wish to determine, in particular, whether apparent differences in illumination in these illusory configurations are reflected in their judgments of dissimilarity.

Before we consider the snake lightness illusion in Experiment 3, we first examine configurations that are analogous to those used in Logvinenko and Maloney (2006), except that viewers in that experiment saw real scenes illuminated by real lights, whereas here they see only pictures that can be interpreted as such scenes.

\section{EXPERIMENT 1}

\section{Method}

We first conducted an experiment in which we asked subjects to scale the dissimilarity between the different targets in different strips and within the same strip using the same techniques and analyses as Logvinenko and Maloney (2006). We tested the hypothesis that the snake lightness illusion is the result of a misinterpretation of the horizontal luminance borders as illumination borders. Under this hypothesis, we expect that targets of different reflectance within the same strip will vary only in the first dimension (surface lightness), whereas targets differing in reflectance but equal in luminance contrast, located in different strips, will vary in the other dimension (surface brightness). Therefore, the hypothesis implies a two-dimensional solution.

Stimuli, subjects, and training. A pattern with three different strips and three different equicontrast targets (Figure 4A) was used as a stimulus display. Three diamonds of different reflectances were rendered as if illuminated by light of three different intensities (pictorial interpretation). The luminance of different patches of the pattern is presented in Table 1 . We index the patches as $i j$, $i, j=1,2,3$, as shown in Figure 4A, and we denote the luminance of target patch $i j$ by $L_{i}^{j}$. We note that targets of equal reflectance on the same backgrounds have equal luminance contrast under different illuminations. Five observers participated to the experiment. They were paid for their participation, and none of them was aware of the purpose of the experiments.

The main experiment was preceded by two preliminary experiments. In these experiments, observers gained experience in making dissimilarity judgments on visual stimuli. In the first one, they were presented with a simplified geographical map (seven black ellipses at various distances from each other) and instructed to estimate distance between all possible pairs in a random order. This experiment continued until, on the basis of each observer's responses, the output
Table 1

Target and Immediate Background Luminances in Experiment 1

\begin{tabular}{lcccc}
\hline & Background & Target 1 & Target 2 & Target 3 \\
\hline Strip 1 & 9.54 & 2.90 & 4.41 & 7.18 \\
Strip 2 & 17.63 & 5.38 & 8.16 & 13.27 \\
Strip 3 & 43.12 & 13.15 & 19.95 & 32.46 \\
\hline
\end{tabular}

Note-The labels of the target patches in Figure 4A are composed of two numbers: The first refers to the target, the second to the strip containing it. For example, the label 13 refers to the luminance of Target 1 in Strip 3, whose luminance is given in this table. All luminances are reported in units of $\mathrm{cd} / \mathrm{m}^{2}$.

MDS configuration effectively coincided with the stimulus map. In the second preliminary experiment, the observers were asked to judge the dissimilarity in grayness between all pairs of 10 gray squares randomly presented on a white background. After a few sessions, all the observers were consistent with a one-dimensional MDS output configuration, with dissimilarity a monotonic function of the squares' reflectances.

Procedure. Observers sat at a distance of $1 \mathrm{~m}$ from the wall, where the stimulus display (printed on A4 paper) was mounted at eye level. The experimenter pointed out one pair of diamonds at a time with a laser pointer, asking the observer to rate dissimilarity between that pair of diamonds by a number on a 30-point scale. Before starting the experiment, observers were shown two extremely dissimilar diamonds and two identical surfaces with identical backgrounds and asked to anchor their scale to these cases, treating them as 29 and 0, respectively (see Logvinenko \& Maloney, 2006, for details). Each of the observers repeated the task 3 times, with different randomized orders of pairing of diamonds in different experimental sessions.

Analysis. For all experiments, we modelled the observers' responses by a linear equation based on the results of Logvinenko and Maloney (2006). We describe the stimulus (Figure 4A) as horizontal strips of unequal illumination containing surfaces that can differ in albedo. We define interpreted differences in illumination and albedo as follows: The target region denoted $i j$ is assigned a log albedo index $R=i$ and a $\log$ illumination intensity $E=j$. The values $R$ are a linear transformation of the actual log albedo that would be assigned to the regions if corresponding targets in each strip were interpreted as surfaces with the same albedo under different illuminations. The values $E$ are a linear transformation of the log light intensities in different strips. These values correspond to light intensity and albedo values measured in standardized units. The rated dissimilarity $D$ between two surface-light pairs $\left(R^{1}, E^{1}\right)$ and $\left(R^{2}, E^{2}\right)$ was modeled as

$$
D=\delta_{E} \Delta E+\delta_{R} \Delta R+\beta_{E} E+\beta_{0}+\varepsilon,
$$

where $\varepsilon$ is additive Gaussian error with mean 0 and standard deviation $\sigma$ and $\Delta E=\left|E^{1}-E^{2}\right|$ and $\Delta R=\left|R^{1}-R^{2}\right|$ correspond to standardized differences in log illumination intensity and log surface albedo, respectively. For example, the difference in log luminance between two surfaces interpreted as having the same albedo but situated in different strips (under different illuminations) would correspond to $\Delta E$ for those two strips. Similarly, the difference in luminance between two surfaces in the same strip would count as $\Delta R$ for those surfaces. The term $E=\left(E^{1}+E^{2}\right) / 2$ is the standardized mean $\log$ illumination across two strips. Since dissimilarity should not depend on which surface light pair is labeled 1 or 2, we use absolute differences to capture this symmetry. We refer to these terms as light intensity difference $(\Delta E)$, surface albedo difference $(\Delta R)$, and absolute light level $(E)$. The first term brought about the differences in surface brightness in Logvinenko and Maloney's experiment, the second, the surface lightness.

With this model, the observer's response is a weighted sum of these factors, a constant term, and additive Gaussian error. If, for example, $\delta_{E}=0$, the observer's judgments of dissimilarity are independent of light intensity difference, a kind of lightness constancy. If $\delta_{R}=0$, the observer's judgments of dissimilarity are independent of surface albedo difference, a highly unlikely outcome. 
A
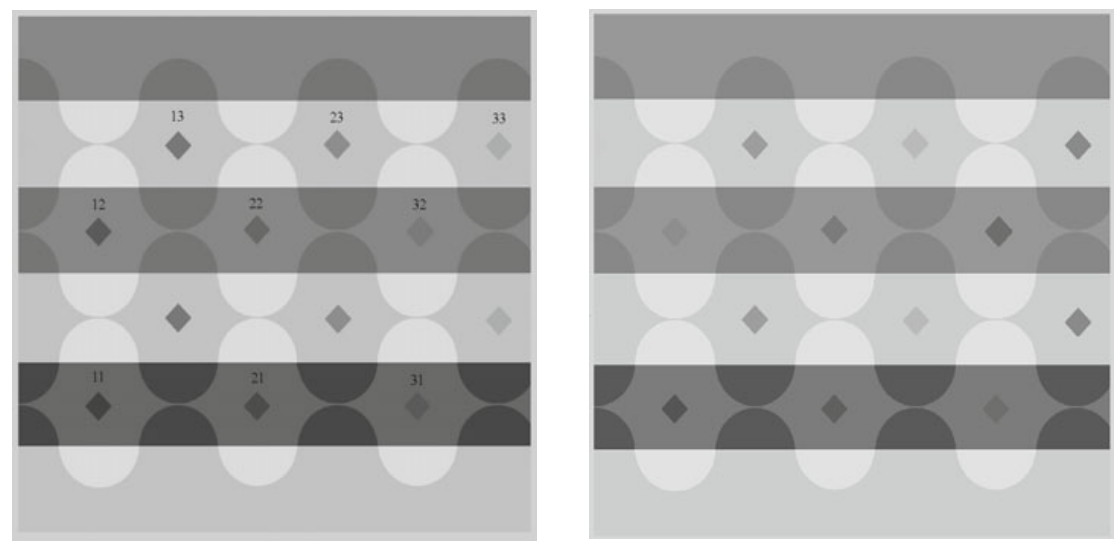

B

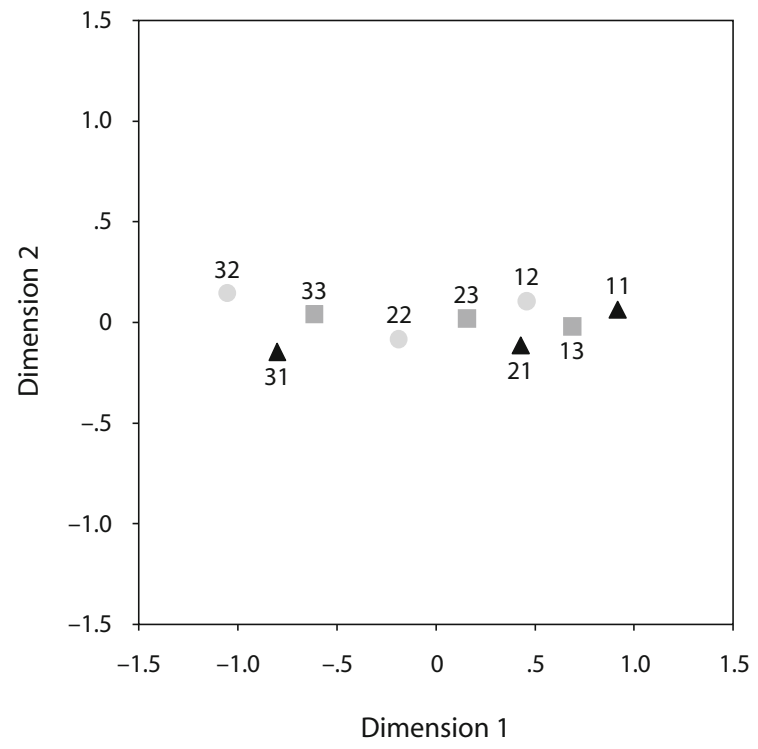

Figure 4. (A) Equicontrast snake patterns. The difference between these is that the test diamonds are arranged in random order in the pattern on the right-hand side (which was actually presented in Experiment 1). The pattern can be interpreted as three different reflectances under three different illuminations. We will refer to "simulated surfaces" under "simulated illuminations" for convenience in describing the stimuli and results; see text. Each point is also labeled. The first number in each label refers to the surface reflectance $(1,2$, and 3$)$ and the second to the strip $(1,2$, and 3$)$. The number in the labels increases in accord with the luminance value of reference (i.e., 1 , lowest luminance; 3, highest luminance). For example, the label 13 refers to the surface of lowest albedo in the strip of highest-intensity simulated illumination. For specification of the actual luminances of parts of the figure, refer to Table 1 . As follows from the table, the local luminance contrast at the border of targets 11, 12, and 13 is the same, as is the local luminance contrast for the targets 21, 22, and 23 (as well as for 31,32 , and 33). Thus, the luminance contrast of the corresponding targets in different strips is equal. (B) The nonmetric multidimensional scaling solution for Experiment 1. Points corresponding to the three simulated reflectances in the strip of lowest-intensity simulated illumination are marked by triangles; those corresponding to the three reflectances in the strip of medium-intensity simulated illumination are marked by circles; those corresponding to the three reflectances in the strip of highest-intensity simulated illumination are marked by squares. The numerical labeling convention is the same as that in Figure 4A. 
Logvinenko and Maloney (2006) found that surface dissimilarity increased with absolute light level. The term with coefficient $\beta_{E}$ allows for this possible effect. The constant term $\beta_{0}$ is of no direct interest, corresponding to the minimum of the arbitrary scale of rated dissimilarities and the standardization of units. We estimated the coefficients of Equation 6 by multiple regression and tested which coefficients are nonzero and report the results of tests of hypotheses that each of the three coefficients $\delta_{E}, \delta_{R}$, and $\beta_{E}$, are 0 . The use of standardized units simplifies the analysis and cannot affect the conclusions drawn from hypothesis tests.

\section{Results}

As expected, we could reject the hypothesis that $\delta_{R}=0$ for all subjects, even with a Bonferroni correction for five tests $(p<.01=.05 / 5)$. This result implies only that subjects' judgments of dissimilarity depended on the difference in surface albedos. The crucial test is whether $\delta_{E}$ is significantly different from zero, indicating that perceived dissimilarity was affected by the difference in perceived illumination in different strips of the illusion configuration. We found that we could not reject the hypothesis that $\delta_{E}=0$ for any of the subjects $(p>.01)$. The third coefficient $\beta_{E}$ captures a minor trend in the data: Pairs of surfaces were perceived as more dissimilar under brighter lights by 2 subjects $(p<.01)$ but not by the remaining 3 . The presence or absence of the effect is irrelevant to the hypothesis under test, but it is an interesting confirmation of one of the results of Logvinenko and Maloney (2006), that the lightness continuum shrank under darker illuminations and expanded under brighter illuminations.

\section{Discussion}

If we accept that observers interpret the long edges in the pattern in Figure 4A as illumination edges, Figure 4A is a pictorial representation of a scene that is very close in structure to the experimental display in Figure 3A used by Logvinenko and Maloney (2006). Contrary to their results, our analysis shows that there is no significant contribution of perceived illumination difference to dissimilarity in Figure 4A. In Figure 4B, we show an MDS solution for the dissimilarity data in Experiment 1 . Again, the difference between Figure 3B and Figure 4B is evident. The points corresponding to surfaces embedded in different strips of the pattern fall close to a straight line. Since the maximal dissimilarity is achieved between the marginal diamonds in the same strip, it is safe to conclude that this line corresponds to the surface lightness dimension. The results of the statistical tests above confirm these conclusions. Therefore, despite the commonly claimed impression that the alternating strips have different apparent illuminations, we find no evidence for a contribution of surface brightness to the dissimilarity between diamonds in adjacent strips. There is an evident "dissociation" between the phenomenological content of the scene and the edge-classification implicit in the dissimilarity ratings, a dissociation not present in the experiment of Logvinenko and Maloney using real surfaces and illuminants.

It must be mentioned, however, that Logvinenko and Maloney (2006) used seven different reflectances, whereas in our experiment there were only three different contrasts.
To ascertain whether this methodological difference is important, the next experiment was carried out.

\section{EXPERIMENT 2}

The purpose of this experiment was to repeat Experiment 1 , using a larger number of diamonds to ascertain whether the results of the previous experiment were due to the small number of stimuli used.

\section{Method}

A pattern with two different strips and five different equicontrast targets (Figure 5A) was used to simulate five diamonds of different reflectances, lit by light of two different intensities. The luminance of different patches of the pattern is presented in Table 2 . The same 5 observers participated in the experiment. The notation and model are as in Experiment 1, but with the surface index $i$ ranging from 1 to 5 , and the illumination index $j$ now ranging from 1 to 2 . The procedure of this experiment was the same as in Experiment 1, except that each subject made five judgments of each pair.

\section{Results and Discussion}

An output MDS configuration for the dissimilarities obtained in Experiment 2 is presented in Figure 5B. The points corresponding to the targets in the different strips have a tendency to merge into a one-dimensional line, as in Figure 4B. We performed the same analysis as in Experiment 1 . The multiple regression fit shows that $\delta_{E}$ is not significantly different from zero for all subjects with a Bonferroni correction for five tests. That is, light differences $\Delta E$ did not affect dissimilarity. As expected, the surface difference (contrast difference) coefficient $\delta_{R}$ was significantly different from 0 for all subjects $(p<.01)$. The results are consistent with those of Experiment 1.

The results of both experiments show that, in fact, there is no patterned shift in dissimilarity that could be due to a perceived difference in illumination of the strips. Yet such a shift exists in the original Adelson's snake pattern, where the diamonds are equiluminant (Figure 1A) rather than equicontrast, as are those used in Experiments 1 and 2. Is this a lightness shift, or surface brightness shift? The following experiment was conducted to answer this question and investigate the snake pattern itself.

\section{EXPERIMENT 3}

The design of this experiment was similar to that of the previous experiment, except that we used equiluminant rather than equicontrast patterns.

Table 2

Target and Immediate Background Luminances in Experiment 2

\begin{tabular}{lcccccc}
\hline & \multicolumn{5}{c}{ Target } \\
\cline { 3 - 7 } & Background & 1 & 2 & 3 & 4 & 5 \\
\hline Strip 1 & 17.63 & 1.89 & 3.08 & 5.38 & 8.16 & 13.27 \\
Strip 2 & 43.12 & 4.63 & 7.54 & 13.15 & 19.95 & 32.46 \\
\hline
\end{tabular}

Note-The labels in Figure 5A are composed of two numbers: The first refers to the target, the second to the strip. For example, the label 12 refers to the luminance of Target 1 in Strip 2, whose luminance is given in this table. All luminances are reported in units of $\mathrm{cd} / \mathrm{m}^{2}$. 
A

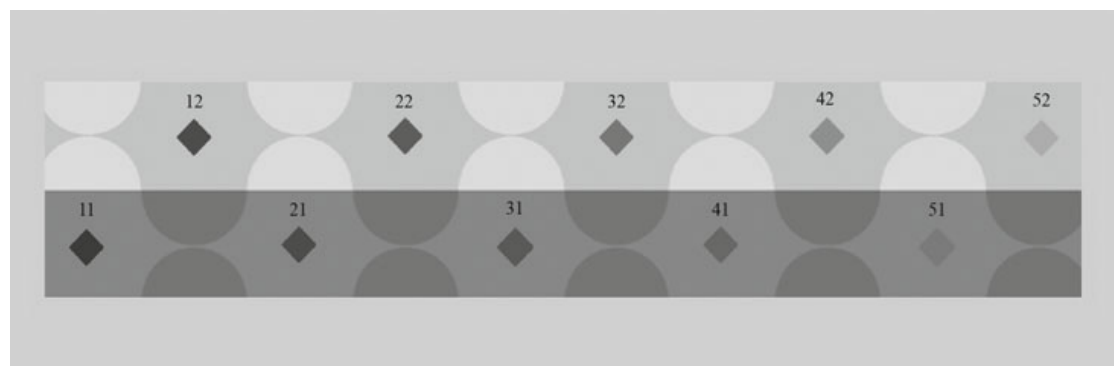

B

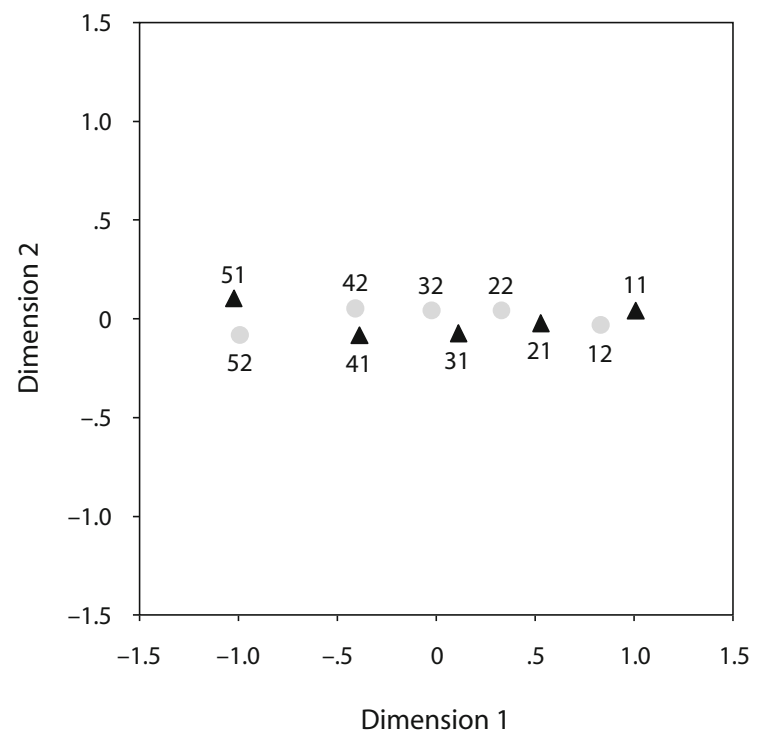

Figure 5. (A) Example of the stimulus pattern for the equicontrast snake patterns used in Experiment 2 . In these patterns, we simulated five different reflectances under two different illuminations. The labeling convention is similar to that used in Figure 4A. In the actual experiment, the positions of the diamonds were randomized from trial to trial. For specification of the actual luminances of parts of the figure, refer to Table 2. (B) The nonmetric multidimensional scaling solution for Experiment 2. Points corresponding to the five simulated reflectances in the strip of lower-intensity simulated illumination are marked by triangles; those corresponding to the five reflectances in the strip of higher-intensity simulated illumination are marked by circles.

\section{Method}

A snake pattern with two different strips and five different equiluminant targets (Figure 6A) was used. The luminance of different patches of the snake pattern is presented in Table 3 . The same $5 \mathrm{ob}-$ servers participated in the experiment. The procedure was the same as in Experiment 2. In setting up the regression model, we confront a problem. We use the indices $i j$, just as in the previous analysis, but now we have no reason to claim that test regions with the same surface albedo index $i$ would be interpreted as having the same albedo. Any constant offset in albedo between corresponding target regions in the two strips will be confounded with a change in illumination. We will return to this point immediately below in interpreting the regression results.

\section{Results and Discussion}

The same regression analysis was performed. Again, the coefficient $\delta_{R}$ corresponding to surface difference (contrast difference) was significantly different from 0 for all subjects $(p<.01)$. However, in contrast with the previous two experiments, the multiple regression fit shows that $\delta_{E}$ is now significantly different from zero for 4 out of 5 subjects, even with a Bonferroni correction for five tests $(p<.01)$. The $p$ value for the remaining subject was less than 0.02 ; that is, the simulated light difference between strips appears to have affected dissimilarity between the diamonds in Figure 6A. As we noted above, however, this difference could also correspond to a shift in interpretation of the albedos from one strip to the other.

The MDS solution in Figure 6B cannot be described as two-dimensional. The significance of the light difference effect means that the points $12,22,32,42$, and 52 (the diamonds in the "light" strip) are shifted rightward along an arc relative to the points $11,21,31,41$, and 51 (the diamonds in the "dark" strip). An increase in simulated 


\section{A}

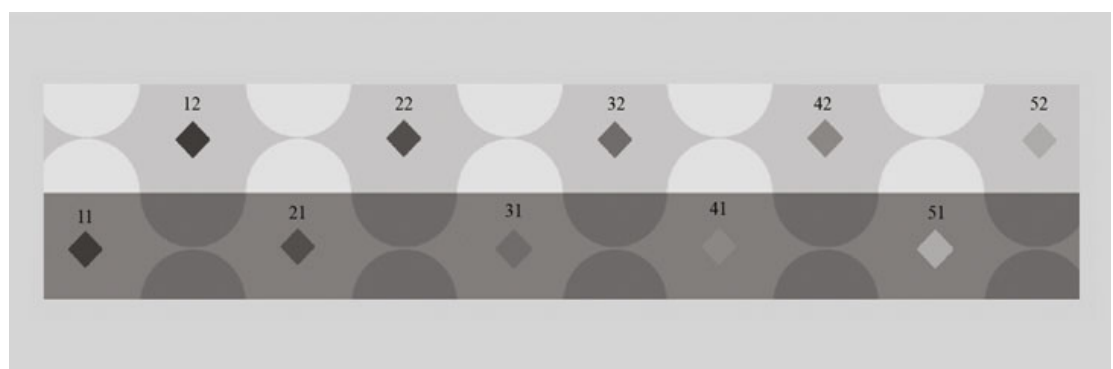

B

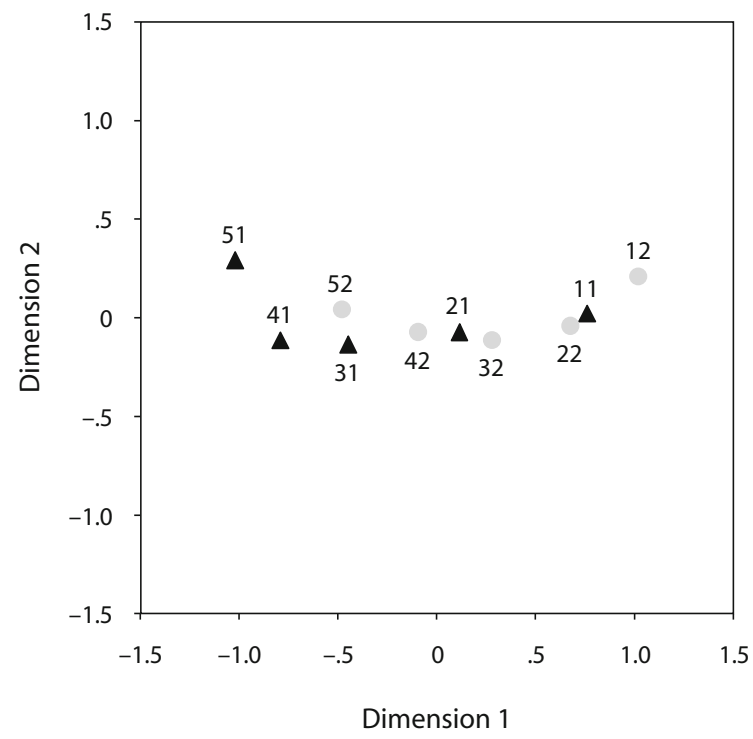

Figure 6. (A) Example of the equiluminant snake stimulus patterns used in Experiment 3. We simulated five different reflectances under two different illuminations. The labeling convention is the same as that used in Figure 5A. The first number in each label increases with the luminance value of the target (i.e., 1 , lowest luminance; 5 , highest luminance). In the actual experiment the positions of the diamonds were randomized from trial to trial. For specification of the actual luminances of parts of the figure, refer to Table 3. As follows from the table, the luminance of the targets 11 and 12 is the same. So is the luminance for the targets 21 and 22 (as well as for 31 and 32, and so on). Thus, the luminance of the corresponding targets in different strips is equal. (B) The nonmetric multidimensional scaling solution for Experiment 3. Points corresponding to the five simulated reflectances in the strip of lower-intensity simulated illumination are marked by triangles, while those corresponding to the five reflectances in the strip of higher-intensity simulated illumination are marked by circles. The pattern is predominantly unidimensional, although curved. There is no clear separation of points corresponding to simulated light intensities that would correspond to a difference in surface brightness; see text.

illumination is perceived as an increase in simulated surface albedo. This overall shift is a surface lightness rather than surface brightness shift. The surface brightness shift would reveal itself as an overall vertical (downward or upward), as in Figure 3B, rather than as a horizontal (rightward) shift.

Note, in conclusion, that it is possible to produce a snake-like pattern with targets that are both equiluminant and equicontrast (Logvinenko, 2002; Logvinenko \&
Ross, 2005). Although such a pattern lacks borders that are readily interpreted as illumination borders, it invokes a lightness illusion stronger than the classic simultaneous lightness contrast effect. In this pattern, not only are the targets equal in luminance, their local surroundings have the same luminance as well. Therefore, the fact that the targets in the classical snake pattern are inserted in the patches of different reflectance is not essential for observing the snake illusion. 
Table 3

Stimulus Patch Luminances in Experiment 3

\begin{tabular}{lcccccc}
\hline & & \multicolumn{5}{c}{ Target } \\
\cline { 3 - 7 } & Background & 1 & 2 & 3 & 4 & 5 \\
\hline Strip 1 & 17.63 & 4.63 & 7.54 & 13.15 & 19.95 & 32.46 \\
Strip 2 & 43.12 & 4.63 & 7.54 & 13.15 & 19.95 & 32.46 \\
\hline
\end{tabular}

Note-The labels in Figure 6A (as well as in Figure 6B) are composed of two numbers: The first refers to the target, the second to the strip. For example, label 12 refers to the luminance of Target 1 in Strip 2, whose luminance is given in this table. The luminance values of the targets in the two strips are identical; only the luminance values of the immediate backgrounds differ. All luminances are reported in units of $\mathrm{cd} / \mathrm{m}^{2}$.

\section{GENERAL DISCUSSION}

The snake illusion, and other illusions of the same sort, such as the tile illusion (Adelson, 1993), have typically been studied using asymmetric matching techniques (Adelson, 1993; Logvinenko, 1999; 2003; Logvinenko et al., 2005; Logvinenko \& Ross, 2005). However, researchers have previously reported that observers do not always achieve complete, satisfactory matches in asymmetric matching experiments involving color or lightness (Brainard, Brunt, \& Speigle, 1997; Foster, 2003; Logvinenko \& Maloney, 2006). The results of Logvinenko and Maloney indicate that perceived dissimilarity is affected by at least two perceptual dimensions corresponding to surface albedo and intensity of illumination. Logvinenko and Maloney referred to these dimensions as surface lightness and surface brightness, and they interpreted previously reported difficulties in setting asymmetric matches as due to the residual component of the surface brightness difference that remains after the observer has made the surface lightness difference as small as possible.

In this article, we used the dissimilarity scaling method of Logvinenko and Maloney to investigate a series of lightness configurations, including the snake illusion (in Experiment 3) We sought to determine how surface lightness and surface brightness contribute to the illusion configurations under consideration. One advantage of our approach over asymmetric matching is that it can give us, in principle, two measures of the component parts of the illusion (i.e., surface lightness and surface brightness). A second advantage is that these measures translate naturally into information about the observer's interpretation of the scene as consisting of one or more regions of uniform illumination ("frameworks"). A third advantage of the dissimilarity scaling method used in the present study is that it does not predetermine the nature of the illusion; that is, one does not have to decide in advance whether the illusion is a lightness or brightness illusion. This method allows one to address this issue experimentally.

Furthermore, in contrast to those who use asymmetrical matching, we did not have to explain to our observers what lightness or brightness is. It is always a difficult task to explain to a naïve observer what one means by a specific "perceptual dimension." We have consistently found that evaluating dissimilarity between the appearances of two differently painted papers (sometimes lit by different lights) was an easy and intuitive task for our observers.
Our analysis shows that observers' dissimilarity judgments of diamond-shaped targets in Figure 1A can be modeled in terms of only one perceptual dimension. More specifically, we found that placing the same diamonds in different strips in Figure 1A resulted in a change in the diamonds' appearance consistent with an effective change in their reflectance. Accordingly, we refer to this dimension as surface lightness.

Therefore, the difference in appearance between the diamonds in the alternating horizontal strips in Figure 1 is predominantly a difference in surface lightness. Although we measured only the appearance of the diamonds, we take this as an indication that there is no surface brightness shift between the horizontal strips as the whole. This interpretation is the physically correct interpretation and all observers were undoubtedly aware that the stimuli were in fact uniformly illuminated. Although the horizontal borders between the strips in the snake pattern (Figure 1) possess cues indicating that they are produced by illumination borders (they are "shadow-compatible," in the terminology of Logvinenko, 2003) our results indicate that they do not contribute to dissimilarity, as an actual illumination border would. We note that, in reproduction, the physical illumination border in Figure 3A may appear indistinguishable from the pictorial illumination border in the snake illusion. In the experimental context, however, the subject had no difficulty in distinguishing real and pictured illumination borders.

Logvinenko (1999) argued that, although the cues to illumination available in the horizontal borders of the snake pattern do not bring about illumination borders in the final percept, these cues might have been taken into account when computing lightness (also see Logvinenko \& Ross, 2005). In other words, when computing surface lightness, the visual system can take a stance that $E^{1} \neq E^{2}$, although it discards this assumption when surface brightness is computed. More specifically, the horizontal luminance borders in the snake pattern contain some features signaling that they are produced by illumination borders (illumination cues) and some signaling that they are produced by reflectance borders (reflectance cues). It is plausible that the luminance contrast is split (in a proportion that depends on the balance of the illumination and reflectance cues) into some amount of lightness contrast and some amount of surface brightness contrast. The stronger the illumination cues, the smaller the amount of the lightness contrast induced by the borders. Since there are other cues indicating that the illumination of the pattern is homogeneous, the surface brightness contrast is blocked and is not perceived. As a result, we see only a reduced amount of lightness contrast at the horizontal borders in Figures $1 \mathrm{~A}$ and $1 \mathrm{~B}$.

Logvinenko et al. (2005) showed that bending the strips in the snake pattern, as in Figure 7, considerably reduces the illusion strength. As can be seen in Figure 7, equicontrast targets $(a)$ and $(b)$ do not seem to have equal lightness, as do the corresponding equicontrast targets in Figure $1 \mathrm{~B}$. We conjecture that this difference is due to the replacement of straight horizontal borders by curved. This change shifts the balance of the cues in favor of classifica- 
tion as a reflectance border and altering the interpretation of the configuration.

We can decrease the luminance contrast of target $c$ in the light strip until it matches target $a$ in the dark strip. This is an additional indication that the apparent crossstrips shift between targets $a$ and $b$ in Figure 7 takes place along the surface lightness dimension, because one can make up for it by changing the surface lightness of the target. If it were a surface brightness shift, such a match would be impossible, as Logvinenko and Maloney (2006) showed. Indeed, it follows from their study that equicontrast targets in differently illuminated frameworks are perceived as different, and this difference cannot be reduced by manipulating the reflectance of either target.

One can appreciate the magnitude of the lightness contrast reduction by comparing Figures $1 \mathrm{~A}$ and $1 \mathrm{~B}$ with Figure $1 \mathrm{C}$. Direct observation shows that the lightness contrast across the horizontal borders in Figures $1 \mathrm{~A}$ and $1 \mathrm{~B}$ is less than the lightness contrast across the snake-curved borders in Figure $1 \mathrm{C}$, despite the fact that the luminance contrasts across both borders are the same, as mentioned above. Indeed, patches in the dark "snakes" in Figure 1C look distinctively darker than do the patches of the same reflectance in the dark strips in Figures 1A and 1B. Likewise, patches in the bright "snakes" in Figure 1C look somewhat lighter

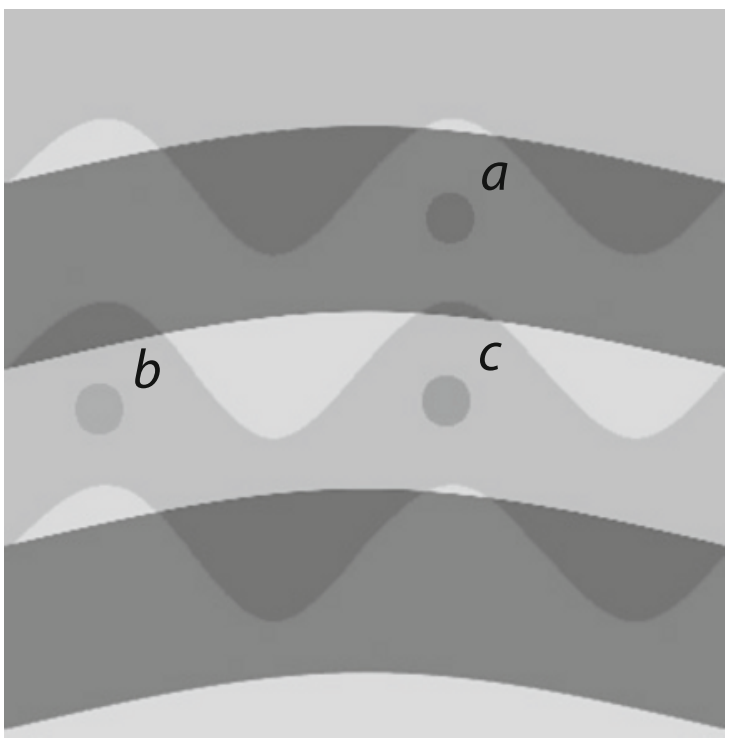

Figure 7. A snake pattern from Logvinenko et al. (2005), with the luminance distribution as in Figure 1B. Being identical to the targets in Figure 1B, $a$ and $b$ are equicontrast, but they appear different, unlike the equicontrast targets in Figure 1B. However, this difference in appearance between equicontrast targets $a$ and $b$ is of a different sort than that between the equicontrast papers in the differently illuminated halves in Figure $3 \mathrm{~A}$. By changing the luminance contrast between the target and background, it is possible to get a lightness match for the targets in different strips although they appear to be lit by different lights. Although physically darker than $b$, target $c$ has about the same lightness as target $a$. Therefore, unlike real papers illuminated by real lights, targets in strips with different pictorial illuminations can match each other. than do the patches of the same reflectance in the bright strips in Figures 1A and 1B. Logvinenko and Ross (2005) observed similar effects in Adelson's (1993) tile pattern. Measuring the lightness not only of the test diamonds in the bright and dark strips, but also of all patches in Adelson's tile pattern, they quantified these differences.

Such an account leads to this prediction: The bigger the luminance contrast across the horizontal borders, the bigger the lightness illusion. This prediction was recently verified experimentally by Petrini and Logvinenko (2005) and Petrini (in press). For example, the horizontal strips in Figure $1 \mathrm{C}$ possess the same shadow cues (e.g., straightness) as in Figure $1 \mathrm{~A}$ and $1 \mathrm{~B}$, but the perception of the targets is veridical (Logvinenko et al., 2005). This is because the luminance ratio across the horizontal borders in Figure $1 \mathrm{C}$ is very close to 1 . We believe that the lightness contrast reduction across the horizontal borders most likely takes place in Figure $1 \mathrm{C}$ as well, but its magnitude is too small to be noticed.

We conjecture that the snake lightness illusion is a specifically pictorial illusion arising from "erroneous" lightness contrast reduction across shadow-compatible luminance borders in the snake pattern. It is worth reminding the reader that when test diamonds are cut off from paper and presented as separate objects "floating" in front of the snake-shaped background, the illusion is very much reduced (Logvinenko \& Kane, 2004). This is in line with the fact that Adelson's tile pattern produces no illusion when implemented as a cardboard wall of blocks (Logvinenko, Kane, \& Ross, 2002).

One might argue that "seeing" transparency or unevenly illuminated strips in the snake pattern (Figure 1A) is a matter of immediate experience. Does this observation contradict our results? We do not believe so. Our results show that, whatever this impression of uneven illumination (or transparency) is, it is of a different kind than that arising from real illumination difference. The latter contributed to dissimilarity judgments in the previous experiment (Logvinenko \& Maloney, 2006), whereas the former did not.

This raises a fundamental methodological question: To what extent can pictures, used as stimuli in experiments on lightness perception, be treated as equivalent to three-dimensional scenes? Color, lightness, and material perception are commonly studied using pictures of threedimensional scenes presented on computer displays, and it is not obvious that the picture is equivalent to viewing the scene depicted (Gibson, 1979; Gombrich, 1960; Kennedy, 1974). It is evident that, under such viewing conditions, depth information is inconsistent. Pictorial depth cues signal a three-dimensional interpretation of the scene, whereas cues such as motion parallax and binocular disparity are consistent with a flat surface embedded in the surface of the screen.

A picture of a three-dimensional scene is evidently ambiguous; it could be interpreted as either a flat image or a three-dimensional scene. Moreover, when the stimulus configuration is very simple, there may be additional interpretations of the scene. Consider, in particular, the snake configuration (Figure 1C). First, the scene may be perceived as flat and uniformly illuminated (the physi- 
cally correct interpretation), or as a picture of a scene. Then, note that either the darker straight stripes, or the dark "snakes," could be treated as transparent (perceived as "transparency regions"). One could interpret either, neither, or both regions as transparency regions, giving a total of four different pictorial interpretations. Moreover, we can interpret the targets as contained in regions differing in illumination and, simultaneously, on backgrounds differing in reflectance, leading to an indefinite number of additional interpretations.

Two key questions are: "How do these multiple, competing interpretations affect psychophysical judgments?" and "Do they affect different visual judgments (dissimilarity, lightness, etc.) differently?"

One possibility, for example, is that the absence of any contribution of surface brightness to dissimilarity could be the result of competing transparency interpretations. If we assume that a transparency interpretation is equivalent to a difference in illumination between a transparency region and adjacent regions, we note that the two transparency regions just described signal changes in illumination that are opposite in sign. Cancellation of apparent illumination difference could then be responsible for the lack of any contribution of surface brightness to dissimilarity.

However, if this competing transparency hypothesis were correct, it would be difficult to explain why the observer saw any change in apparent illumination of the regions of the snake configuration, or perceived any illusion in apparent lightness. At a minimum, we would need to postulate that the visual system had "dissociated" (Milner \& Goodale, 1995): Judgments of dissimilarity are affected by competing transparency interpretations, but judgments of lightness or apparent illumination are not.

Similarly, if the visual system interprets targets as simultaneously under different illuminations and on different backgrounds, the same surface under a brighter light will appear darker; against a darker background, it will appear lighter. In this case, the visual system is interpreting edges as both illumination edges and surface material edges, a possibility we raised in the introductory section. Again, we might expect cancellation, but any cancellation that might be responsible for a lack of contribution of surface brightness to dissimilarity should also reduce or eliminate the snake illusion itself, unless the visual system had dissociated, and different judgments were based on different interpretations of the scene.

There are many pairs of cues in the snake configuration that signal conflicting information. It is plausible that these conflicts would lead to dissociations in visual judgments, where the visual system uses one set of cues to arrive at one interpretation in making one sort of judgment (similarity), and different sets of cues to arrive at a conflicting interpretation in making the second visual judgment (lightness).

Yet, despite all of the possible interpretations of the snake pattern, the perception of lightness in the snake configuration is remarkably stable. The snake illusion can be observed, and it remains at the same strength when printed on paper, presented on a computer display, or projected on screen. Its strength does not seem to be essentially af- fected by alteration of the size and shape of its patches, or of observation distance. All this appears to be in line with our main result: None of these pictorial interpretations affects lightness perception of the snake pattern.

There exist illusory geometric configurations that pose analogous problems of interpretation. We draw the reader's attention to an analogous finding in the literature on pictorial illusions of length in Wolfe, Maloney, and Tam (2005), who concluded that illusory distortions of perceived line length cannot be attributed to a single depth interpretation of the pictured scene. It is also plausible that the visual system has no consistent interpretation of such pictured scenes.

Although the study of pictorial illusions certainly gives insight about how the visual system analyzes lightness and color, there is reason to believe that, in everyday threedimensional scenes, there is more relevant information about the light field in a scene, and therefore less ambiguity in classifying illumination and material edges (Boyaci, Doerschner, Snyder, \& Maloney, 2006; Maloney, 1999, 2002). It is plausible that the visual system will arrive at a consistent interpretation of illumination and surface layout in those scenes that is not too far from the actual. The ability of the visual system to interpret pictures is well worth study, but should not distract from, or be confused with, the problem of estimating lightness and color in everyday scenes.

\section{AUTHOR NOTE}

This work was supported by an EPSRC Grant EP/C010353/1 (A.D.L.) and NIH/NEI EY08226 (L.T.M.). Correspondence concerning this article should be addressed to A. D. Logvinenko, Department of Vision Sciences, Glasgow Caledonian University, Cowcaddens Road, Glasgow G40BA, Scotland (e-mail: a.logvinenko@gcal.ac.uk).

\section{REFERENCES}

Adelson, E. H. (1993). Perceptual organization and the judgment of brightness. Science, 262, 2042-2044.

AdELSON, E. H. (2000). Lightness perception and lightness illusion. In M. Gazzaniga (Ed.), The new cognitive neurosciences ( 2 nd ed., pp. 339-351). Cambridge, MA: MIT Press.

Blakeslee, B., \& McCourt, M. E. (2003). A multiscale spatial filtering account of brightness phenomena. In L. Harris \& M. Jenkin (Eds.), Levels of perception (pp. 47-72). New York: Springer.

Boyaci, H., Doerschner, K., Snyder, J. L., \& Maloney, L. T. (2006). Surface color perception in three-dimensional scenes. Visual Neuroscience, 23, 311-321.

Brainard, D. H., Brunt, W. A., \& Speigle, J. M. (1997). Color constancy in the nearly natural image: I. Asymmetric matches. Journal of the Optical Society of America A, 14, 2091-2110.

Cavanagh, P., \& Leclerc, Y. G. (1989). Shape from shadows. Journal of Experimental Psychology: Human Perception \& Performance, 15, 3-27.

Churma, M. E. (1994). Blue shadows: Physical, physiological, and psychological causes. Applied Optics, 21, 4719-4722.

CoRnsweet, T. N. (1970). Visual perception. New York: Academic Press.

Cox, T. F., \& Cox, M. A. A. (2001). Multidimensional scaling (2nd ed.). Boca Raton, FL: Chapman \& Hall/CRC.

Fine, I., MacLeod, D. I. A., \& Boynton, G. M. (2003). Surface segmentation based on luminance and color statistics of natural scenes. Journal of the Optical Society of America A, 20, 1283-1291.

Foster, D. H. (2003). Does colour constancy exist? Trends in Cognitive Sciences, 7, 439-443.

GiBson, J. J. (1979). The ecological approach to visual perception. Boston: Houghton Mifflin.

GiLchrist, A. [L.] (2006). Seeing black and white. New York: Oxford University Press. 
Gilchrist, A. [L.], Delman, S., \& Jacobsen, A. (1983). The classification and integration of edges as critical to the perception of reflectance and illumination. Perception \& Psychophysics, 33, 425-436.

Gilchrist, A. [L.], Kossyfidis, C., Bonato, F., Agostini, T., CatalIOTTI, J., Li, X., ET AL. (1999). An anchoring theory of lightness perception. Psychological Review, 106, 795-834.

Gombrich, E. H. (1961). Art and illusion: A study in the psychology of pictorial representation (2nd ed.). Oxford: Pantheon Press.

Heggelund, P. (1974). Achromatic color vision: I. Perceptive variables of achromatic colors. Vision Research, 14, 1071-1079.

Kardos, L. (1934). Ding und Schatten [Thing and shadow]. Zeitschrift für Psychologie, 23, 1-184.

KATZ, D. (1935). The world of colour. Oxford: Kegan Paul

KenNedy, J. M. (1974). A psychology of picture perception. Oxford: Jossey-Bass.

Kingdom, F. A. A. (2003). Color brings relief to human vision. Nature Neuroscience, 6, 641-644.

KoffKa, K. (1935). Principles of gestalt psychology. New York: Harcourt, Brace.

LAND, E. H., \& MCCANN, J. J. (1971). Lightness and retinex theory. Journal of the Optical Society of America, 61, 1-11.

Logvinenko, A. D. (1999). Lightness induction revisited. Perception, 28, 803-816.

LogVinenKo, A. D. (2002). The anchoring effect in lightness perception in humans. Neuroscience Letters, 334, 5-8.

Logvinenko, A. D. (2003). A fair test of the effect of a shadowincompatible luminance gradient on the simultaneous lightness contrast (followed by discussion). Perception, 32, 717-730.

Logvinenko, A. D. (2005). Does luminance contrast determine lightness? Spatial Vision, 18, 337-345.

Logvinenko, A. D., Adelson, E. H., Ross, D. A., \& Somers, D. (2005) Straightness as a cue for luminance edge interpretation. Perception \& Psychophysics, 67, 120-128.

LogVinenKo, A. D., \& KANE, J. (2004). Hering's and Helmholtz's types of simultaneous lightness contrast. Journal of Vision, 4, 1102-1110.

Logvinenko, A. D., KAnE, J., \& Ross, D. (2002). Is lightness induction a pictorial illusion? Perception, 31, 73-82

Logvinenko, A. D., \& Maloney, L. T. (2006). The proximity structure of achromatic surface colors and the impossibility of asymmetric lightness matching. Perception \& Psychophysics, 68, 76-83.

Logvinenko, A. D., \& Menshikova, G. (1994). Trade-off between achromatic colour and perceived illumination as revealed by the use of pseudoscopic inversion of apparent depth. Perception, 23 1007-1023.

Logvinenko, A. D., \& Ross, D. A. (2005). Adelson's tile and snake illusions: A Helmholtzian type of simultaneous lightness contrast. Spatial Vision, 18, 25-72.

Maloney, L. T. (1999). Physics-based approaches to modeling surface color perception. In K. R. Gegenfurtner \& L. T. Sharpe (Eds.), Color vision: From genes to perception (pp. 387-422). Cambridge: Cambridge University Press

MaLONEY, L. T. (2002). Illuminant estimation as cue combination. Journal of Vision, 2, 493-504.

MARR, D. (1982). Vision: A computational investigation into the human representation and processing of visual information. San Francisco: W. H. Freeman.

Milner, A. D., \& Goodale, M. A. (1995). The visual brain in action. Oxford: Oxford University Press.

Párraga, C. A., Troscianko, T., \& Tolhurst, D. J. (2000). The human visual system is optimised for processing the spatial information in natural visual images. Current Biology, 10, 35-38.

Petrini, K. (in press). Multiplicative and additive Adelson's snake illusions. Perception.
Petrini, K., \& Logvinenko, A. D. (2005). The additive and multiplicative component contribution into Adelson's snake lightness illusion. Perception, 34 (ECVP Abstract Supplement), 103.

Rock, I. (1975). An introduction to perception. New York: Macmillan.

Rubin, J. M., \& Richards, W. A. (1982). Color vision and image intensities: When are changes material? Biological Cybernetics, 45, 215-226.

Somers, D. C., \& AdELson, E. H. (1997). Junctions, transparency and brightness. Investigative Ophthalmology \& Visual Science (Supplement), 38, S453.

WALLACH, H. (1948). Brightness constancy and the nature of achromatic colors. Journal of Experimental Psychology, 38, 310-324.

Whittle, P. (1994a). The psychophysics of contrast brightness. In A. L. Gilchrist (Ed.), Lightness, brightness and transparency (pp. 35-110). Hillsdale, NJ: Erlbaum.

Whittle, P. (1994b). Contrast brightness and ordinary seeing. In A. L. Gilchrist (Ed.), Lightness, brightness and transparency (pp. 111-158). Hillsdale, NJ: Erlbaum.

Whittle, P. (2003). Contrast colours. In R. Mausfeld \& D. Heyer (Eds.), Colour perception: Mind and the physical world (pp. 115-138). New York: Oxford University Press.

Wolfe, U., Maloney, L. T., \& TAM, M. (2005). Distortions of perceived length in the frontoparallel plane: Tests of perspective theories. Perception \& Psychophysics, 67, 967-979.

\section{NOTES}

1. See also Figure 1B in Logvinenko and Ross (2005), where the same can be observed for a similar illusion which will be referred to as the tile lightness illusion (Adelson, 1993).

2. Logvinenko and Ross (2005) measured all three illusions for the same observers and found that, on average, the snake and tile illusions are 6.5 and 5 times as strong as the simultaneous contrast effect, respectively.

3. The luminance ratio rule should not be confused with Wallach's (1948) luminance ratio principle, which involves the luminance of a target and the luminance of its immediate background. Both rules predict the same result for targets surrounded by regions that are equal in luminance.

4. Note that the equiluminant targets have luminance contrasts of different signs along the borders with their immediate surrounds (one target is incremental and the other is decremental). Contrary to the general belief that it is impossible to equate incremental and decremental targets in appearance (e.g., Heggelund, 1974; Whittle, 1994a, 1994b) the equiluminance diamonds in Figure 1C look remarkably similar.

5. The patches were arranged radially around a single hexagonal patch in order to permit ready comparison of results with these scenes to results in three-dimensional scenes where the patches were the sides and top of a frustrum of a cone with hexagonal cross section. We will not refer to this second experiment in the following.

6. Note, however, that Logvinenko and Maloney (2006) reject a Euclidean rule of combination in favor of the additive city-block metric implicit in Equation 6. The spatial configuration illustrates the highly separable effects of illumination and surface change, but Euclidean distance within the MDS configuration does not accurately represent dissimilarity. The city-block metric of Equation 6 does accurately represent dissimilarity. See Logvinenko and Maloney (2006).

7. The reader should not confuse surface brightness as measured by the method of Logvinenko and Maloney (2006), with the term brightness as used in the vision literature, or confuse surface lightness with "lightness." The terms surface brightness and surface lightness refer to the dimensions derived from dissimilarity data via the method of Logvinenko and Maloney (2006)

(Manuscript received June 8, 2007; revision accepted for publication January 30, 2008.) 